\title{
ON TRICOMI'S RELATION FOR THE HILBERT TRANSFORMATION
}

\author{
by P. G. ROONEY
}

(Received 20th July, 1974)

1. Introduction. Tricomi [2] has shown that if $\phi_{i} \in L_{p i}(-\infty, \infty), i=1,2$, where $1<p_{i}<\infty,\left(p_{1}\right)^{-1}+\left(p_{2}\right)^{-1}<1$, and if $H$ denotes the Hilbert transformation, that is

$$
(H f)(x)=\pi^{-1}(P) \int_{-\infty}^{\infty} f(t)(t-x)^{-1} d t
$$

where the symbol $(P)$ denotes that the integral is taken in the Cauchy principal value sense, then

$$
H\left(\phi_{1} H \phi_{2}+\phi_{2} H \phi_{1}\right)=\left(H \phi_{1}\right)\left(H \phi_{2}\right)-\phi_{1} \phi_{2} .
$$

When one first looks at (2), one would naturally think of using the Fourier transformation to prove it, making use of the well-known formulas for the Fourier transform of $H f$ and for the Fourier transform of a product. However, difficulties with a proof along this line soon become apparent, for the condition $\left(p_{1}\right)^{-1}+\left(p_{2}\right)^{-1}<1$, which is needed for the left hand side of (2) to exist, implies that at least one $p_{i}$ is larger than two, and consequently the corresponding $\phi_{i}$ may not have a Fourier transform. Indeed Tricomi's proof used the theory of $H_{p}$-spaces in the upper half-plane as outlined in $[1, \S 5.12]$.

In this paper we shall show that a proof of (2) in which the Fourier transformation plays the crucial role can be given. This seems worthwhile both from considerations of simplicity, and since the proof of the principal theorem in $[1, \S 5.12$, Theorem 103], that Tricomi used is only given incompletely there.

In $\S 2$ we shall define some notations and prove some preliminary lemmas, while in $\S 3$ we give our proof of (2).

2. Notations and preliminary lemmas. We shall denote the Fourier transformation by $\mathscr{F}$; that is if $f \in L_{1}(-\infty, \infty)$, then

$$
(\mathscr{F} f)(x)=(2 \pi)^{-1 / 2} \int_{-\infty}^{\infty} e^{i x t} f(t) d t .
$$

It is well known that $\mathscr{F}$ can be extended to $L_{p}(-\infty, \infty)$ for $1 \leqq p \leqq 2$, as a bounded operator from $L_{p}$ to $L_{p^{\prime}}$ where now and henceforth

$$
p^{-1}+\left(p^{\prime}\right)^{-1}=1
$$

indeed on $L_{2}(-\infty, \infty) \mathscr{F}$ is unitary. The image of $f$ under $\mathscr{F}$, the Fourier transform, will be denoted by $\hat{f}$. 
If $\phi_{1}$ and $\phi_{2}$ are locally integrable on $(-\infty, \infty)$, their convolution, $\phi_{1} * \phi_{2}$, is defined, for all $x$ for which the integral exists, by

$$
\phi_{1} * \phi_{2}(x)=(2 \pi)^{-1 / 2} \int_{-\infty}^{\infty} \phi_{1}(x-t) \phi_{2}(t) d t
$$

One relation between the Fourier transformation and convolution is given by the following lemma.

Lemma A. If $\phi_{i} \in L_{2}(-\infty, \infty), i=1,2$, then

$$
\left(\phi_{1} \phi_{2}\right)^{\wedge}=\hat{\phi}_{1} * \hat{\phi}_{2} \text {. }
$$

Proof. The result is equivalent to [1, Theorem 65].

As noted earlier, we shall be using the formula for the Fourier transform of $H f$. We state this as a lemma.

LEMmA B. If $f \in L_{2}(-\infty, \infty)$, then

$$
(H f)^{\wedge}(x)=-i \operatorname{sgn} x f^{\wedge}(x) .
$$

Proof. This is proved in the course of the proofs of [1, Theorems 90 and 91].

Formula (2) involves the product of functions from different $L_{p}$ spaces. Concerning such products we have the following lemma.

Lemma C. Suppose that $\phi_{i} \in L_{p_{1}}(-\infty, \infty), i=1,2$, where $\left(p_{1}\right)^{-1}+\left(p_{2}\right)^{-1} \leqq 1$, and let $p^{-1}=\left(p_{1}\right)^{-1}+\left(p_{2}\right)^{-1}$. Then $\phi_{1} \phi_{2} \in L_{p}(-\infty, \infty)$, and

$$
\left\|\phi_{1} \phi_{2}\right\|_{p} \leqq\left\|\phi_{1}\right\|_{p_{1}}\left\|\phi_{2}\right\|_{p_{2}} \text {. }
$$

Proof. An easy application of Holder's inequality.

One final lemma will be rather basic in our argument.

Lemma D. Suppose that $f_{1} \in L_{1}(-\infty, \infty), f_{2} \in L_{2}(-\infty, \infty)$ and $\hat{f}_{1}=\hat{f}_{2}$ a.e. Then $f_{1}=f_{2}$ a.e.

Proof. From [1, Theorem 14], the integral $(2 \pi)^{-1 / 2} \int_{-\infty}^{\infty} e^{-i x y} \hat{f}_{1}(y) d y$ is $(\mathrm{C}, 1)$ summable to $f_{1}$ a.e., and from [1, Theorem 59], the integral $(2 \pi)^{-1 / 2} \int_{-\infty}^{\infty} e^{-i x y} \hat{f}_{2}(y) d y$ is $(\mathrm{C}, 1)$ summable to $f_{2}$ a.e. But since $\hat{f}_{1}=\hat{f}_{2}$ a.e., the two integrals are the same, and hence $f_{1}=f_{2}$ a.e.

3. Tricomi's Theorem. We can now prove the main result.

THEOREM. If $\phi_{l} \in L_{p_{l}}(-\infty, \infty)$, where $1<p_{l}<\infty,\left(p_{1}\right)^{-1}+\left(p_{2}\right)^{-1}<1$, then (2) holds. 
Proof. Suppose first that $\phi_{1}$ and $\phi_{2}$ are continuous functions with compact support, and call the right hand side of $(2) f_{1}$ and the left hand side $f_{2}$. We shall show that $f_{1}$ and $f_{2}$ satisfy the hypotheses of Lemma D.

Since $\phi_{i} \in L_{2}$, and $H$ is a bounded operator from $L_{p}$ to $L_{p}$ for $1<p<\infty$ [1, Theorem 101], $H \phi_{i} \in L_{2}$, and hence by Lemma C, $\left(H \phi_{1}\right)\left(H \phi_{2}\right)$ and $\phi_{1} \phi_{2} \in L_{1}$, so that $f_{1} \in L_{1}$. Since $\phi_{1}$ is bounded, $\phi_{1} H \phi_{2}$ is in $L_{2}$, as is $\phi_{2} H \phi_{1}$, and thus since $H$ is a bounded operator from $L_{2}$ to $L_{2}$, and since $f_{2}=H\left(\phi_{1} H \phi_{2}+\phi_{2} H \phi_{1}\right)$, we have $f_{2} \in L_{2}$.

But from Lemmas $\mathrm{A}$ and $\mathrm{B}$,

$$
\begin{aligned}
\hat{f}_{1}(x) & =\left(H \phi_{1}\right)^{\wedge} *\left(H \phi_{2}\right)^{\wedge}(x)-\hat{\phi}_{1} * \hat{\phi}_{2}(x) \\
& =(2 \pi)^{-1 / 2} \int_{-\infty}^{\infty}\left(\left(H \phi_{1}\right)^{\wedge}(x-t)\left(H \phi_{2}\right)^{\wedge}(t)-\hat{\phi}_{1}(x-t) \hat{\phi}_{2}(t)\right) d t \\
& =-(2 \pi)^{-1 / 2} \int_{-\infty}^{\infty} \hat{\phi}_{1}(x-t) \hat{\phi}_{2}(t)(\operatorname{sgn}(x-t) \operatorname{sgn} t+1) d t .
\end{aligned}
$$

Now if $x>0$,

while if $x<0$,

$$
\operatorname{sgn}(x-t) \operatorname{sgn} t+1=\left\{\begin{array}{l}
0, t<0 \\
2,0<t<x \\
0, t>x
\end{array},\right.
$$

Hence, for almost all $x$,

$$
\operatorname{sgn}(x-t) \operatorname{sgn} t+1=\left\{\begin{array}{l}
0, t<x \\
2, x<t<0 . \\
0, t>0
\end{array}\right.
$$

$$
\begin{aligned}
\hat{f}_{1}(x) & =-(2 / \pi)^{1 / 2} \begin{cases}\int_{0}^{x} \hat{\phi}_{1}(x-t) \hat{\phi}_{2}(t) d t, & x>0 \\
\int_{x}^{0} \hat{\phi}_{1}(x-t) \hat{\phi}_{2}(t) d t, & x<0\end{cases} \\
& =-(2 / \pi)^{1 / 2} \operatorname{sgn} x \int_{0}^{x} \hat{\phi}_{1}(x-t) \hat{\phi}_{2}(t) d t .
\end{aligned}
$$

Also, from Lemma $\mathrm{B}$, for almost all $x$,

$$
\hat{f}_{2}(x)=-i \operatorname{sgn} x\left(\phi_{1} H \phi_{2}+\phi_{2} H \phi_{1}\right)^{\wedge}(x) .
$$

But since $\phi_{i}$ and $H \phi_{i} \in L_{2}$, from Lemma A,

$$
\begin{aligned}
\hat{f}_{2}(x) & =-i \operatorname{sgn} x\left(\hat{\phi}_{1} *\left(H \phi_{2}\right)^{\wedge}(x)+\left(H \phi_{1}\right)^{\wedge} * \hat{\phi}_{2}(x)\right) \\
& =-i \operatorname{sgn} x(2 \pi)^{-1 / 2} \int_{-\infty}^{\infty}\left(\hat{\phi}_{1}(x-t)\left(H \phi_{2}\right)^{\wedge}(t)+\left(H \phi_{1}\right)^{\wedge}(x-t) \hat{\phi}_{2}(t) d t\right. \\
& =-(2 \pi)^{-1 / 2} \operatorname{sgn} x \int_{-\infty}^{\infty} \hat{\phi}_{1}(x-t) \hat{\phi}_{2}(t)(\operatorname{sgn} t+\operatorname{sgn}(x-t)) d t
\end{aligned}
$$


But if $x>0$,

while if $x<0$

$$
\operatorname{sgn} t+\operatorname{sgn}(x-t)=\left\{\begin{array}{l}
0, t<0 \\
2,0<t<x \\
0, t>x
\end{array},\right.
$$

Hence

$$
\operatorname{sgn} t+\operatorname{sgn}(x-t)=\left\{\begin{array}{l}
0, t<x \\
-2, x<t<0 \\
0, t>0
\end{array}\right.
$$

$$
\begin{aligned}
\hat{f}_{2}(x) & =-(2 / \pi)^{1 / 2} \operatorname{sgn} x\left\{\begin{array}{l}
\int_{0}^{x} \hat{\phi}_{1}(x-t) \hat{\phi}_{2}(t) d t, \quad x>0 \\
-\int_{x}^{0} \hat{\phi}_{1}(x-t) \hat{\phi}_{2}(t) d t, \quad x<0
\end{array}\right. \\
& =-(2 / \pi)^{1 / 2} \operatorname{sgn} x \int_{0}^{x} \hat{\phi}_{1}(x-t) \hat{\phi}_{2}(t) d t=\hat{f}_{1}(x) \text { a.e. }
\end{aligned}
$$

Thus by Lemma $\mathrm{D}, f_{1}=f_{2}$ a.e., and (2) is true if $\phi_{1}$ and $\phi_{2}$ are continuous functions with compact support.

Now each side of (2) represents a bounded linear transformation from $L_{p l}, i=1,2$, to $L_{p}$. For if the norm of the Hilbert transformation on $L_{r}$ is denoted by $M_{r}$, then from Lemma $\mathrm{C}$, since $p>1$

$$
\begin{aligned}
& \left\|H\left(\phi_{1} H \phi_{2}+\phi_{2} H \phi_{1}\right)\right\|_{p} \leqq M_{p}\left\|\phi_{1} H \phi_{2}+\phi_{2} H \phi_{1}\right\|_{p} \\
& \leqq M_{p}\left(\left\|\phi_{1} H \phi_{2}\right\|_{p}+\left\|\phi_{2} H \phi_{1}\right\|_{p}\right) \leqq M_{p}\left(\left\|\phi_{1}\right\|_{p_{1}}\left\|H \phi_{2}\right\|_{p_{2}}+\left\|H \phi_{1}\right\|_{p_{1}}\left\|\phi_{2}\right\|_{p_{2}}\right) \\
& \leqq M_{p}\left(M_{p_{1}}+M_{p_{2}}\right)\left\|\phi_{1}\right\|_{p_{1}}\left\|\phi_{2}\right\|_{p_{2}}
\end{aligned}
$$

and

$$
\begin{aligned}
& \left\|\left(H \phi_{1}\right)\left(H \phi_{2}\right)-\phi_{1} \phi_{2}\right\|_{p} \leqq\left\|\left(H \phi_{1}\right)\left(H \phi_{2}\right)\right\|_{p}+\left\|\phi_{1} \phi_{2}\right\|_{p} \\
& \leqq\left\|H \phi_{1}\right\|_{p_{1}}\left\|H \phi_{2}\right\|_{p_{2}}+\left\|\phi_{1}\right\|_{p_{1}}\left\|\phi_{2}\right\|_{p_{2}} \\
& \leqq\left(M_{p_{1}} M_{p_{2}}+1\right)\left\|\phi_{1}\right\|_{p_{1}}\left\|\phi_{2}\right\|_{p_{2}} .
\end{aligned}
$$

Suppose then that $\phi_{1} \in L_{p_{1}}$, while $\phi_{2}$ is continuous with compact support. Then there is a sequence. $\phi_{1, n}$ of continuous functions with compact support which converge in $L_{p_{1}}$ to $\phi_{1}$. Denoting limits in $L_{p}$ by $\mathscr{L}_{p}$, we have

$$
\begin{aligned}
& H\left(\phi_{1} H \phi_{2}+\phi_{2} H \phi_{1}\right)=\mathscr{L}_{p} H\left(\phi_{1, n} H \phi_{2}+\phi_{2} H \phi_{1, n}\right) \\
& =\mathscr{L}_{p}\left(\left(H \phi_{1, n}\right)\left(H \phi_{2}\right)-\left(\phi_{1, n}\right)\left(\phi_{2}\right)\right)=\left(H \phi_{1}\right)\left(H \phi_{2}\right)-\left(\phi_{1}\right)\left(\phi_{2}\right),
\end{aligned}
$$

so that (2) is true if $\phi_{1} \in L_{p_{1}}$, and $\phi_{2}$ is continuous with compact support. 
Finally, if $\phi_{i} \in L_{p_{i}}, i=1,2$, where $\left(p_{1}\right)^{-1}+\left(p_{2}\right)^{-1}<1$, then there is a sequence $\phi_{2, n}$ of continuous functions with compact support converging in $L_{p_{2}}$ to $\phi_{2}$. Hence

$$
\begin{aligned}
& H\left(\phi_{1} H \phi_{2}+\phi_{2} H \phi_{1}\right)=\mathscr{L}_{p} H\left(\phi_{1} H \phi_{2, n}+\left(\phi_{2, n}\right)\left(H \phi_{1}\right)\right) \\
& =\mathscr{L}_{p}\left(\left(H \phi_{1}\right)\left(H \phi_{2, n}\right)-\left(\phi_{1}\right)\left(\phi_{2, n}\right)\right)=\left(H \phi_{1}\right)\left(H \phi_{2}\right)-\phi_{1} \phi_{2},
\end{aligned}
$$

and our result is proved.

\section{REFERENCES}

1. E. C. Titchmarsh, Theory of Fourier integrals, Second Edition (Oxford, 1948).

2. F. G. Tricomi, On the finite Hilbert transformation, Quart. J. Math. (2) 2, 1951, 199-211 (see also F. G. Tricomi, Integral equations, Interscience Publ. (New York, 1957), § 4.3).

\section{UNIVERSITY OF TORONTO}

